\title{
Blunt Traumatic Intraperitoneal Bladder Rupture After Alcohol Intake That Responds to Non-Operative Conservative Management
}

\author{
Kenji Ohgaki ${ }^{\mathrm{a}, \mathrm{b}}$, Masatoshi Sakamoto ${ }^{\mathrm{a}}$
}

\begin{abstract}
Blunt traumatic intraperitoneal bladder rupture is usually treated by surgical repair. We report a patient with intraperitoneal bladder rupture secondary to blunt abdominal trauma that responded to nonoperative conservative management using a transurethral catheter. A 58-year-old woman was admitted to our Department of Internal Medicine complaining of lower abdominal pain and urinary retention after drinking alcohol. Computed tomography (CT) scan revealed collection of fluid in the abdominal cavity and fresh blood clots in the bladder. At 2 days after admission, she was moved from the Department of Internal Medicine to Urology. Cystoscopy revealed an old hematoma in the bladder. She complained of abdominal pain when the Foley catheter was removed. At 3 days after admission, cystoscopy revealed a small tear at the bladder dome, which led to a definitive diagnosis of intraperitoneal bladder rupture. At 4 days after admission, a CT scan detected only a small amount of fluid in the abdominal cavity. Conservative therapy was continued because urine could be constantly drained. At 2 weeks after admission, the bladder rupture had healed.
\end{abstract}

Keywords: Blunt trauma; Intraperitoneal bladder rupture; Conservative management

\section{Introduction}

Intraperitoneal bladder rupture can occur due to a sudden rise in intravesical pressure following a blow to the lower abdomen in a patient with a distended bladder. This increase in pressure results in rupture of the weakest portion of the bladder, such as the dome, where the bladder is in contact with the peritoneal surface. In adults, watertight repair is required for intraperitoneal bladder rupture with major hemorrhage secondary to blunt abdominal trauma, using decompression with

Manuscript submitted November 12, 2018, accepted November 26, 2018

${ }^{a}$ Department of Urology, Asao General Hospital, Kanagawa 215-0021, Japan ${ }^{b}$ Corresponding Author: Kenji Ohgaki, Department of Urology, Asao General Hospital, 6 Chome-25-1 Kamiasao Asao Ward, Kawasaki, Kanagawa 2150021, Japan. Email: kogaki2018@gmail.com

doi: https://doi.org/10.14740/wjnu380 a urethral catheter or suprapubic tube [1]. In children, initial non-operative treatment can be applied for isolated intraperitoneal bladder rupture without improper bladder drainage and prolonged urinary drainage [2]. Herein, we report successful non-operative management of an adult patient with blunt traumatic intraperitoneal bladder rupture.

\section{Case Report}

A 58-year-old woman fell on the way home after drinking alcohol for $5 \mathrm{~h}$, and presented to the emergency room (ER) with lower abdominal pain and urinary retention. On examination, she was afebrile $\left(35.0^{\circ} \mathrm{C}\right)$, with a blood pressure of $96 / 68 \mathrm{~mm}$ $\mathrm{Hg}$ and a pulse rate of 52 beats per minute (BPM). Oxygen saturation was $94 \%$ in room air. Laboratory tests revealed a white blood cell (WBC) count of $14,940 / \mathrm{mm}^{3}$, hemoglobin $13.2 \mathrm{~g} /$ $\mathrm{dL}$, serum C-reactive protein (CRP) $0.05 \mathrm{mg} / \mathrm{dL}$, blood urea nitrogen (BUN) $30.0 \mathrm{mg} / \mathrm{dL}$, serum creatinine $1.88 \mathrm{mg} / \mathrm{dL}$, and gross hematuria. Non-contrast abdominopelvic computed tomography (CT) revealed a large amount of ascites (Fig. 1a) and fresh blood clots in an intra-distended bladder (Fig. 1b). Based on these findings, she was diagnosed with post-renal renal failure due to urinary retention, bladder tamponade due to bladder cancer hemorrhage, and ascites due to cancerous peritonitis.

To improve her general condition, she was admitted to the Department of Internal Medicine from the ER. On the day of admission, lower abdominal pain and gross hematuria continued, even under pain control and with the use of an indwelling Foley catheter. On the next day, she was moved to the Department of Urology. On examination, she was febrile $\left(38.4{ }^{\circ} \mathrm{C}\right)$, with a blood pressure of $114 / 73 \mathrm{~mm} \mathrm{Hg}$, and a pulse rate of 70 BPM. Laboratory tests revealed a WBC count of $8,270 / \mathrm{mm}^{3}$, hemoglobin $11.1 \mathrm{~g} / \mathrm{dL}$, serum CRP $3.25 \mathrm{mg} / \mathrm{dL}$, BUN $19.0 \mathrm{mg} /$ $\mathrm{dL}$, serum creatinine $0.71 \mathrm{mg} / \mathrm{dL}$, and gross hematuria. There was a large hematoma in the bladder under cystoscopy. The Foley catheter was removed because no fresh hemorrhage was apparent. A urethral catheter was placed because she complained of severe lower abdominal pain at night.

Three days after admission, cystoscopy revealed a small perforation on the right side of the bladder dome (Fig. 2a). The patient was diagnosed with intraperitoneal bladder rupture, and a renal pelvic balloon catheter was placed after cystoscopy. Four days after admission, a second non-contrast CT scan detected loss of fluid in the abdominal cavity (Fig. 1c). Five days 


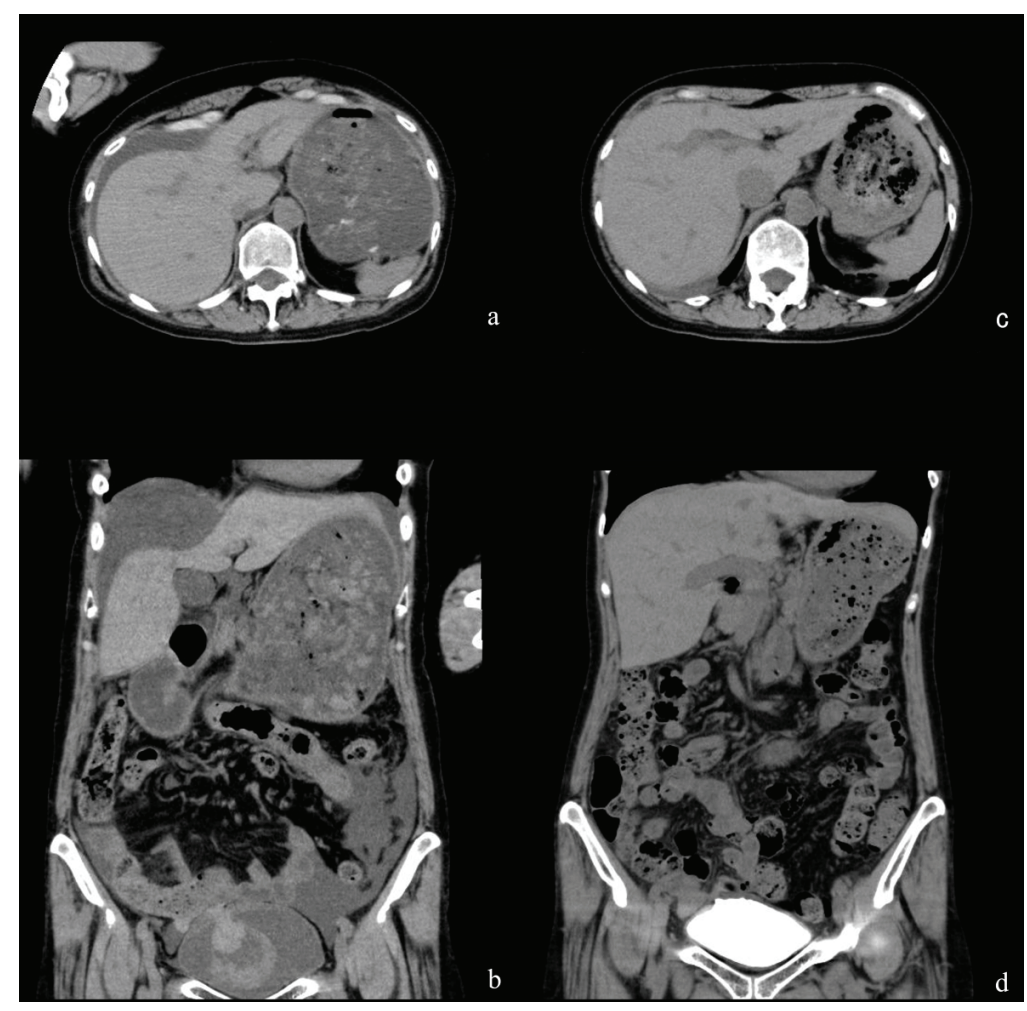

Figure 1. Findings from CT. (a) Non-contrast CT at the upper abdominal level showed a large amount of ascites at admission. (b) Non-contrast CT with coronal reconstruction at the urinary bladder level showed fresh blood clots in the bladder at admission. (c) Non-contrast CT at the upper abdominal level showed a small amount of ascites at 4 days after admission. (d) A CT cystogram with coronal reconstruction at the urinary bladder level showed no extravasation of contrast agent from the bladder dome at 14 days after admission.

after admission, a cystogram showed extravasation of contrast agent from the bladder dome (Fig. 3). Conservative management was continued because urine could be constantly drained and her general condition and laboratory data had improved.

Eleven days after admission, cystoscopy showed no perforation at the bladder dome (Fig. 2b), but a papillary tumor was found on the left side of the perforation scar (Fig. 2c). We performed a cold punch biopsy because cytology findings indicated a class IIIb tumor. The pathological findings showed papillary cystitis. Fourteen days after admission, a third CT cystogram showed no extravasation of contrast agent (Fig. 1d). The patient was discharged in good condition because she could urinate after urethral catheter removal. Eleven days after discharge, cystoscopy revealed no perforation or papillary tumor (Fig. 2d), indicating that the bladder rupture had healed under conservative non-operative management.

\section{Discussion}

A blow to the lower abdomen in a patient with a distended bladder may result in rupture of the weakest part of the bladder due to the increased intravesical pressure. The bladder dome is in contact with the peritoneal surface and is a weak point that is susceptible to rupture. An alcohol-intoxicated patient may also have decreased sensation of an abnormal behavioral response to bladder filling, and this increases the risk of bladder rupture. The volume of ingested alcohol and the resulting diuretic effect increase bladder filling. This then causes bladder distension that may produce an atonic decompensated bladder that is so stretched and thinned that rupture can result from minor trauma [3].

Gross hematuria is a characteristic found in bladder rupture, and the patient may also complain of suprapubic pain or difficulty or inability to urinate. Tenderness in the suprapubic area and absence of bowel sounds may be found, especially if the rupture is intraperitoneal [4]. Laboratory tests may suggest that intraperitoneal bladder rupture is due to oliguric acute renal failure because reabsorption of urea and creatinine through the peritoneum elevates the levels of urea and creatinine in the blood [5]. CT cystography has advantages over plain film cystography in evaluation of suspected bladder rupture due to blunt trauma [6].

In adults, the bladder is protected from injury by the bony pelvis and lies below the level of the obliterated hypogastric arteries when empty. As the bladder fills, the fundus distends laterally and the dome, covered by the peritoneum, rises into the abdominal cavity and is free to move. In children, the bladder is almost entirely in the abdominal organ, and the peritoneum covers the fundus as far as the level of the bladder neck in newborns [1].

Non-operative management can occasionally be applied 


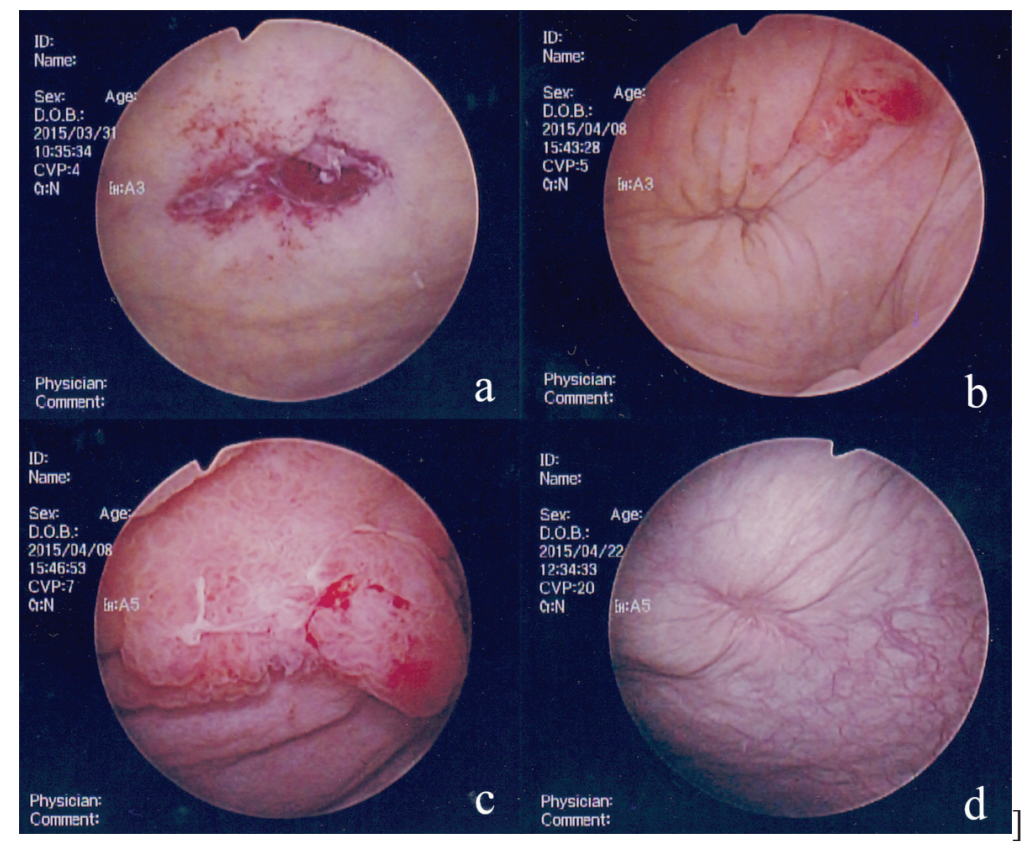

Figure 2. Findings from cystoscopy. (a) A small tear with blood clots at the bladder dome at 3 days after admission. (b) An obstructed tear and a papillary tumor on the left side of the tear at 11 days after admission. (c) An enlarged papillary tumor at 11 days after admission. (d) Healed bladder rupture at 11 days after discharge.

for extraperitoneal vesical rupture but intraperitoneal perforations of the bladder must be explored surgically [7]. Intraperitoneal bladder rupture is usually severe and unlikely to heal spontaneously, and may result in fatal peritonitis [8]. Osman et al suggested that non-operative treatment, including adequate bladder drainage, intravenous antibiotics and percutaneous intraperitoneal drainage, was an initial option for isolated intraperitoneal bladder rupture in children. Indications for surgical intervention include improper bladder drainage, prolonged

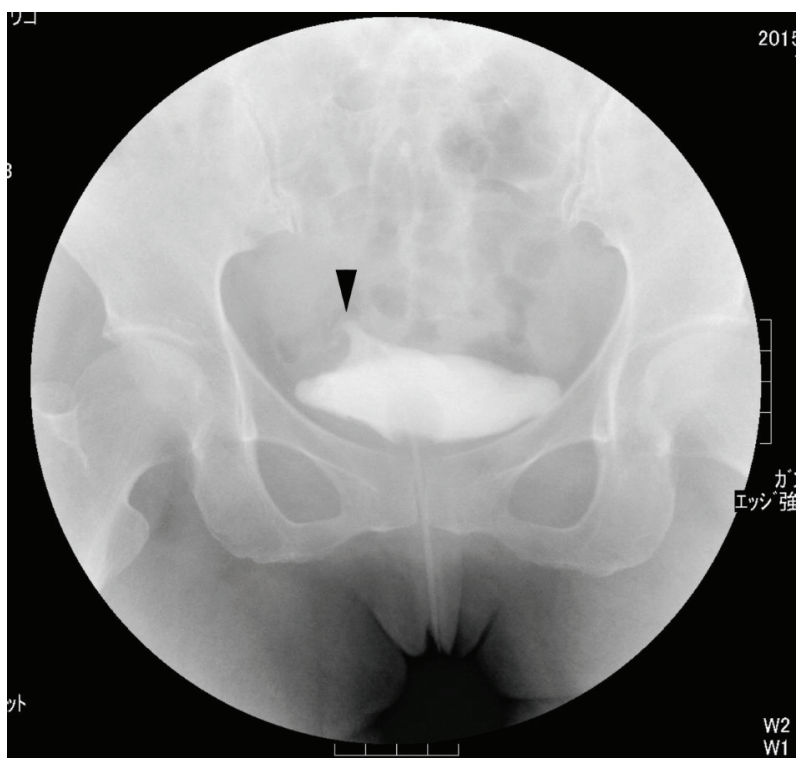

Figure 3. A conventional cystogram showed extravasation of contrast agent from the bladder dome (arrowhead) at 5 days after admission. urinary drainage through the peritoneal drain, and a lack of clinical or laboratory improvement [2].

In our patient, CT and laboratory tests revealed severe gross hematuria, a large amount of fluid in the abdominal cavity, and renal failure. The patient was treated conservatively because she was initially diagnosed with gross hematuria, cancerous ascites, and post-renal renal failure due to urinary tract malignancy. Post-renal renal failure due to urinary retention was diagnosed because renal function was normal with Foley catheterization. Bladder cancer hemorrhage was suggested because cystoscopy revealed a large hematoma at the bladder dome. However, the course of the patient's disease was reevaluated because the cause of lower abdominal pain could not be determined. She fell on her way home in an inebriated state, and she had bruised her hands and knees, but she did not have a memory of a bruised abdomen. The patient had sudden abdominal pain after the fall and requested an ambulance through a passer-by. Clinical examinations revealed abrasions to her hands and knees, and tenderness in the suprapubic region. Bladder rupture was suspected because the lower abdominal pain has been reduced by renal pelvic balloon catheterization 3 days after the injury. Identification of a small tear at the bladder dome finally led to a definitive diagnosis.

Intraperitoneal bladder rupture should generally be repaired urgently. In our case, conservative treatment was continued due to an initial misdiagnosis. Fortunately, gross hematuria, infection, renal failure, and lower abdominal pain were controlled by conservative treatment. CT revealed no extravasation from the bladder with transurethral catheterization, and CT cystography and cystoscopy revealed spontaneous healing of the bladder rupture. Therefore, in this case, conservative treatment of intraperitoneal bladder rupture was successful for 
the following reasons: 1) The tear was small and confined to the bladder dome; 2) The urine was not infected; 3) The urine was constantly drained by a renal pelvic balloon catheter and no intraperitoneal fluid was observed; 4) There was no longterm hemorrhage from the bladder rupture; and 5) There were no other abdominal organ injuries or fractures.

\section{Conclusions}

The outcome of this case suggests that conservative therapy can be used as an initial approach for an isolated blunt traumatic intraperitoneal bladder rupture. Indications for non-operative management include no extravasation from the bladder in constant drainage, control of infection and gross hematuria, and improvement of clinical and laboratory data.

\section{Conflict of Interest}

The authors declare that there is no conflict of interest associated with the work in the manuscript.

\section{Financial Support}

No funding was received for the publication.

\section{Abbreviations}

ER: emergency room; BPM: beats per minute; WBC: white blood cell; CRP: C-reactive protein; BUN: blood urea nitrogen; CT: computed tomography

\section{References}

1. Corriere JN, Jr., Sandler CM. Bladder rupture from external trauma: diagnosis and management. World J Urol. 1999;17(2):84-89.

2. Osman Y, El-Tabey N, Mohsen T, El-Sherbiny M. Nonoperative treatment of isolated posttraumatic intraperitoneal bladder rupture in children-is it justified? J Urol. 2005;173(3):955-957.

3. Herd AM, Crofts NG, Lee LM, Girard JV, Sweetland RJ. Isolated bladder rupture after minor trauma in a patient with alcohol intoxication. J Emerg Med. 1994;12(3):409411.

4. Corriere JN, Jr., Sandler CM. Management of the ruptured bladder: seven years of experience with 111 cases. J Trauma. 1986;26(9):830-833.

5. Heyns CF, Rimington PD. Intraperitoneal rupture of the bladder causing the biochemical features of renal failure. Br J Urol. 1987;60(3):217-222.

6. Deck AJ, Shaves S, Talner L, Porter JR. Computerized tomography cystography for the diagnosis of traumatic bladder rupture. J Urol. 2000;164(1):43-46.

7. Lucey DT, Smith MJ, Koontz WW, Jr. Modern trends in the management of urologic trauma. J Urol. 1972;107(4):641-646.

8. Hsieh CH, Chen RJ, Fang JF, Lin BC, Hsu YP, Kao JL, Kao YC, et al. Diagnosis and management of bladder injury by trauma surgeons. Am J Surg. 2002;184(2):143147. 